\title{
Investigating the Role of Adducts in Protein Supercharging with Sulfolane
}

\author{
Kevin Aart Douglass, Andre R. Venter
}

Department of Chemistry, Western Michigan University, 1901 W. Michigan Ave., Kalamazoo, MI 49008-5413, USA

\begin{abstract}
The supercharging effect of sulfolane on cytochrome $c$ (cyt $c$ ) during electrospray ionization mass spectrometry (ESI-MS) in the absence of conformational effects was investigated. The addition of sulfolane on the order of $1 \mathrm{mM}$ or greater to denaturing solutions of cyt $c$ results in supercharging independent of protein concentration over the range of 0.1 to $10 \mu \mathrm{M}$. While supercharging was observed in the positive mode, no change in the charge state distribution was observed in the negative mode, ruling out polarity-independent factors such as conformational changes or surface tension effects. A series of sulfolane adducts observed with increasing intensity concurrent with increasing charge state suggests that a direct interaction between sulfolane and the charged sites of cyt $c$ plays an important role in supercharging. We propose that charge delocalization occurring through large-scale dipole reordering of the highly polar supercharging reagent reduces the electrostatic barrier for proximal charging along the cyt $c$ amino acid chain. Supporting this claim, supercharging was shown to increase with increasing dipole moment for several supercharging reagents structurally related to sulfolane.
\end{abstract}

Key words: Supercharging, Multiple charging, Sulfolane, Cytochrome $c$, Cyt $c$, Electrospray, Adduct, Protein, New model, Dipole, Gas-phase basicity, Apparent gas phase basicity, Basic site, Charge stabilization

\section{Introduction}

$\mathrm{E}^{\mathrm{s}}$ lectrospray ionization mass spectrometry (ESI-MS) is a powerful tool to study biologically relevant macromolecules such as proteins, owing in part to its ability to generate multiply charged ions. The ESI process begins with the formation of highly charged droplets containing the analyte of interest. As the droplets evaporate, the Coulombic repulsion between charges on the surface eventually overcomes the surface tension at a point called the Rayleigh limit, resulting in a Coulombic fission event [1, 2]. Many small, highly charged progeny droplets containing analyte leave the parent droplet with a disproportionately large fraction of the surface charge [3]. These progeny droplets

Electronic supplementary material The online version of this article (doi:10.1007/s13361-011-0319-1) contains supplementary material, which is available to authorized users.

Correspondence to: Andre R.Venter; e-mail: andre.venter@wmich.edu undergo subsequent evaporation and fission, and this process repeats until only gas-phase ions remain.

The actual mechanism of ion formation in ESI is believed to occur along one of two pathways, the ion evaporation model [4-6] (IEM) or the charge residue model [7] (CRM). There is strong evidence that large molecules such as proteins are predominantly charged by the CRM process [8-10] while smaller molecules are primarily charged by the IEM process $[11,12]$. According to the CRM, the solvent evaporation and successive fission events result in the formation of progeny droplets containing a single analyte molecule, which is then charged through a charge transfer process between the charge carriers on the surface of the droplet and the analyte molecule upon final droplet evaporation [7].

When a protein is analyzed by ESI-MS, several peaks corresponding to different charge states are observed. This is referred to as the charge state distribution (CSD) or envelope. For a given set of experimental conditions, each protein will acquire a characteristic highest observed charge state. HOCS 
(highest observed charge state) was used in place of the more established $\mathrm{CS}_{\max }$ (maximum charge state) to remove any ambiguity between the charge state with the highest intensity (referred to as HICS in the text) and the charge state with the highest charge. Early work by Loo et al. [13] and Covey et al. [14] suggested that the HOCS of a protein is determined by its number of basic residues. Several years later, using an argument similar to Wong et al. to describe the maximum charging of polyethylene glycols (PEGs) [15], Schnier et al. extended this theory by suggesting that the HOCS of proteins should also be limited by the electrostatic repulsion between protonated basic residues [16]. According to this model, the charges on the surface of an electrosprayed droplet at the moment of final evaporation are partitioned between the solvent and the protein based on differences in their apparent gas-phase basicities. The apparent gas-phase basicity of each basic site decreases with increasing charge state due to Coulombic repulsion from existing charges [17, 18]. Therefore, the HOCS should be the last charge state in which an open basic site has a gas-phase basicity above that of the solvent. Schnier et al. were able to predict the HOCS for 13 proteins within an average of $6 \%$ of the experimental values [16].

For folded proteins, both the entire envelope and the HOCS are generally shifted to lower charge states (higher $\mathrm{m} / \mathrm{z}$ ) than for unfolded ones. While charging limited by apparent gas-phase basicities agrees well for unfolded proteins, the maximum charge state obtained by ESI-MS for many folded proteins has been observed to fall below that predicted by apparent gasphase basicities [19]. Several studies instead show a correlation between the surface area of the protein and the maximum charge state $[8,10,20-22]$. These results suggest that at least for folded proteins, the maximum charge state is determined by the Rayleigh limit for a droplet the same size as the protein. The Rayleigh limit is given by the equation $\mathrm{q}^{2}=64 \pi^{2} \varepsilon \sigma \mathrm{r}^{3}$ Equation (1) where $q$ is the charge on the droplet, $\varepsilon$ is the permittivity of free space, $\sigma$ is the surface tension, and $r$ is the droplet radius. The difference between these models for unfolded and folded proteins can be reconciled by considering that compact folded protein structures might result in a final droplet size too small to accommodate the number of charges for all basic sites to become charged by the CRM process [19] and that some prospective charge carrying sites may be inaccessible, buried inside the folded protein.

An alternative model explaining the conformational dependence of CSDs has been suggested in which the multiple-charging of folded proteins does not appear to be restricted by the Rayleigh limit [22-25], but rather by the neutralization of opposite charges [25-28]. Both acidic and basic residues can be charged in solution depending on solution $\mathrm{pH}$ and their respective $\mathrm{pK}_{\mathrm{a}}$ values, and the net charge of a protein is the sum of the positive and negative charges. According to the conformation-dependent neutralization (CDN) theory [27], zwitterionic states might be more stable in folded conformations than in unfolded ones. This additional stability could alter the gas-phase basicity so that neutralization of charges opposite to that of the ESI polarity does not occur during the ESI process, shifting the CSD to lower values for folded proteins.

In practice, the extent of multiple charging for proteins in ESI-MS is dependent on several experimental parameters. Solvent composition [9, 29-33] determines charge availability according to the surface tension and Rayleigh limit and can impose an upper limit on charging based on gas-phase basicity. Denaturing solutions, such as those with high acid, base, or alcohol content, result in CSDs shifted to higher charge states due to protein unfolding [34-36]. Interactions with other molecules in the gas phase, whether as part of the solvent system or introduced, can shift CSDs to higher [37] or lower $[19,38]$ charge states due to charge transfer reactions. Some instrumental settings [13, 23, 39-42] such as source voltages [13], sprayer tip diameter [42], and gas pressures [23, 40] can also shift the CSD.

There is a special case of CSD modification called "supercharging" where small amounts of additives such as m-nitrobenzyl alcohol [32] or sulfolane [33] are added to electrosprayed solutions, usually proteins, which results in a dramatic enhancement of multiple charging. Increased multiple charging of proteins has many benefits, such as reducing the mass range required for protein analysis as well as improved top-down protein sequencing [43, 44]. First reported in 2000 [45], supercharging has been the subject of considerable debate.

Initial results from the Williams' group, obtained with denatured proteins in acidified solution, suggested a rough relationship between the surface tension of the supercharging reagent and the average charge state [9,31]. The supercharging reagents are thought to enrich in the droplet during solvent evaporation owing to their low volatility, concomitantly increasing the surface tension of the droplet. This increased surface tension would increase charge availability according to the Rayleigh limit theory. However, subsequent work from Grandori's group indicated that in the absence of conformational changes, multiple charging is largely independent of surface tension [23-25], a result that was later supported by Loo et al. [46].

Recently, possibly due to increasing interest in using ESIMS as a tool for top-down proteomics and for studying protein interactions, most of the reported supercharging research has been on proteins in the native state. In 2009, Loo et al. determined that supercharging of noncovalent protein complexes from aqueous solutions does not appear to be caused by conformational changes in solution [46]. However, soon afterwards Williams et al. showed similarities between results obtained from thermal denaturation and from supercharging of aqueous solutions, suggesting that supercharging from aqueous solutions is due to conformational changes in the electrospray droplets resulting from droplet heating [47]. It was proposed that more extended protein conformations result in a final droplet size with larger charge availability according to the Rayleigh limit theory. While several studies have since appeared that support the role of conformational changes occurring late 
during ESI as a potential source of supercharging in aqueous solutions [48-50], other studies have questioned this model $[37,46,51]$

There is still a good deal of uncertainty that surrounds the supercharging phenomenon. While some evidence points to an increase in the surface tension of mature electrospray droplets, [9] several studies support alternative causes for the supercharging of proteins $[24,25,46,52]$. In this paper we attempt to study the contribution of effects other than conformational changes to supercharging by investigating the role of bulk solvent properties such as dipole and chemical effects including protein - sulfolane adduct formation.

\section{Experimental}

\section{Chemicals}

Bovine cytochrome $c$ (cyt $c$ ), sulfolane, sulfolene, and dimethyl sulfoxide were purchased from Sigma-Aldrich (St. Louis, MO, USA), LC-MS grade methanol and formic acid were purchased from EMD Chemicals (Darmstadt, Germany), and water was obtained from Fluka. 3-Chlorothietane-1,1-dioxide (CAS 15953-83-0) was purchased from Synthonix (Wake Forest, $\mathrm{NC}$, USA). All chemicals were used as received without further purification.

\section{Sample Preparation}

Stock solutions of cyt $c$ were prepared in water followed by dilution to the specified concentration and solvent composition of 50:50:0.1 MeOH: $\mathrm{H}_{2} \mathrm{O}$ :formic acid (FA) unless otherwise noted. Samples were prepared daily at room temperature and were analyzed immediately following preparation. For samples containing supercharging reagents, aqueous solutions containing the supercharging reagent were added as part of the water fraction of the cyt $c$ samples.

\section{ESI-MS}

For electrospray ionization experiments, a linear ion trap mass spectrometer (LTQ; Thermo Scientific, Waltham, MA, USA) equipped with the Ionmax ESI source was used. The MS was tuned by automated routine on the $m / z 941(+13$ charge state) peak of the cyt $c$ envelope in the positive mode and $\mathrm{m} / z 1746$ (7 charge state) in the negative mode. Instrumental parameters are given in Table 1. The position of the sprayer tip relative to the mass inlet was optimized manually for the optimum signal stability and intensity. Initially, relatively mild ionization conditions were chosen to retain adducts during ion formation (parameter set $\mathrm{A}$ in Table 1). For comparing positive and negative mode supercharging, as well as comparing several supercharging reagents, the sheath gas and capillary temperature were increased to reduce adduct formation and give cleaner spectra (parameter set B in Table 1). Samples were introduced by infusion and spectra were obtained by spectral averaging with 1 min acquisition times using automatic gain
Table 1. Instrumental Parameters used During ESI-MS. Parameters Promoting Adduct Retention are Presented in Column A. Parameters in Column B were used to Compare the Multiple Charging of cyt $c$ in the Positive and Negative Modes

\begin{tabular}{lccc}
\hline Parameter & \multirow{2}{*}{ A } & \multicolumn{2}{c}{ B } \\
\cline { 3 - 4 } & & Positive & Negative \\
\hline Capillary temperature $\left({ }^{\circ} \mathrm{C}\right)$ & 180 & 270 & 270 \\
Capillary voltage $(\mathrm{V})$ & +43 & +43 & -43 \\
Tube lens $(\mathrm{V})$ & +110 & +110 & -250 \\
Spray voltage $(\mathrm{kV})$ & +5 & +3.5 & -3.5 \\
Solvent flow rate $(\mu \mathrm{L} / \mathrm{min})$ & 5 & 5 & 5 \\
Sheath gas $($ arb. units) & 5 & 25 & 25 \\
\hline
\end{tabular}

control. Trap fill times were typically 1 to $10 \mathrm{~ms}$. Protein charge states were determined using the formula [14] $q_{i}=\frac{m_{i-1}+1}{m_{i-1}-m_{i}}$ Equation (2) where $q_{i}$ is the charge state of the $i$ th charge state, $\mathrm{m}_{\mathrm{i}}$ is the mass-to-charge ratio of the $i$ th charge state, and $m_{i-1}$ is the mass-to-charge ratio of the $i$ th-1 charge state. The average charge state $q_{a v g}$ was determined using the formula [53] $q_{\text {avg }}=\frac{\sum_{i}^{N} q_{i} W_{i}}{\sum_{i}^{N} W_{i}}$ Equation (3) where $N$ is the total number of protein charge states observed in the mass spectrum and $W_{i}$ is the signal intensity of the $i$ th charge state.

\section{Results and Discussion}

The alcohol and $\mathrm{pH}$ induced denaturing of cyt $c$ were previously studied $[34,54,55]$. In the present solvent system of 50:50:0.1 MeOH: $\mathrm{H}_{2} \mathrm{O}: \mathrm{FA}$, cyt $c$ is expected to obtain an expanded, highly helical conformation. The ESI-MS of $10 \mu \mathrm{M}$ cyt $c$ in Figure 1a shows a bimodal distribution mostly centered on the +15 charge state, confirming that the protein is primarily in the denatured state. Figure 1b shows the shift in the CSD from an average of +15 to +21 with the addition of $300 \mathrm{mM}$ sulfolane to $10 \mu \mathrm{M}$ cyt $c$. Of the several known supercharging reagents, sulfolane was chosen for this study because it showed the highest supercharging ability in a recent publication by Lomeli et al. [33] and is miscible with water. Several peaks corresponding to charged sulfolane products were observed. Figure 1c shows the low $\mathrm{m} / \mathrm{z}$ range of the spectrum of a typical $10 \mu \mathrm{M}$ cyt $c$ sample to which $300 \mu \mathrm{M}$ sulfolane was added. A base peak at $m / z 121$ is observed, corresponding to $[\mathrm{M}+\mathrm{H}]^{+}$, where $\mathrm{M}$ is sulfolane, as well as peaks at $\mathrm{m} / z 153$ and $\mathrm{m} / \mathrm{z} 241$, corresponding to $\left[\mathrm{M}+\mathrm{CH}_{3} \mathrm{OH}+\mathrm{H}\right]^{+}$and $[2 \mathrm{M}+\mathrm{H}]^{+}$, respectively.

Figure 2 shows the change in average charge state (ACS) for several concentrations of cyt $c$ with the addition of increasing amounts of sulfolane. High concentrations of supercharging reagents are required to observe any significant supercharging effect. For known reagents, it has been demonstrated that maximum supercharging is achieved when the concentration of the reagent is in the millimolar range [32,33], well above the micromolar analyte concentration typical in ESI-MS. In the present study, no supercharging was observed for cyt $c$ until the sulfolane concentration of the sample was on the order of $1 \mathrm{mM}$ or higher. It appears that the onset of supercharging depends on 

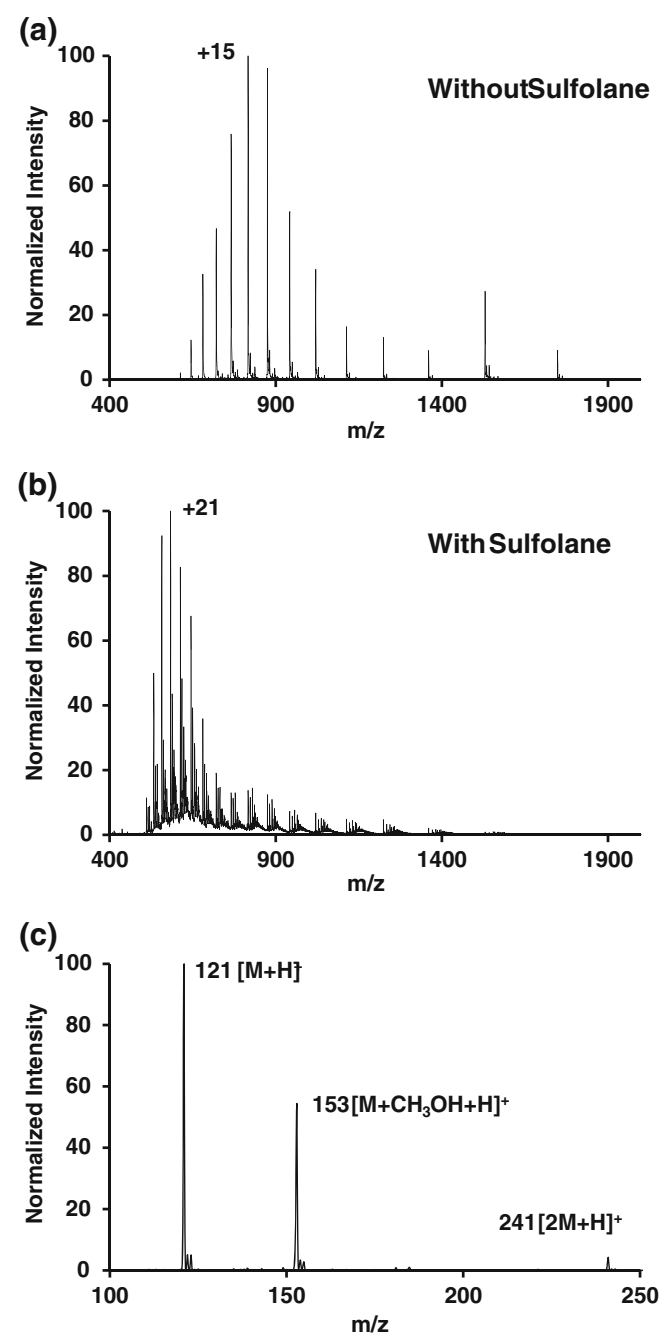

Figure 1. (a) $10 \mu \mathrm{M}$ cyt $c$ in 50:50:0.1 MeOH: $\mathrm{H}_{2} \mathrm{O}: \mathrm{FA}$. (b) $10 \mu \mathrm{M}$ cyt $c$ in 50:50:0.1 MeOH: $\mathrm{H}_{2} \mathrm{O}: \mathrm{FA}$ with $300 \mathrm{mM}$ sulfolane added. (c) Charged sulfolane (M) species were observed when sulfolane was added to cyt $c$ samples. Peak assignment was verified by MS/MS (data not shown)

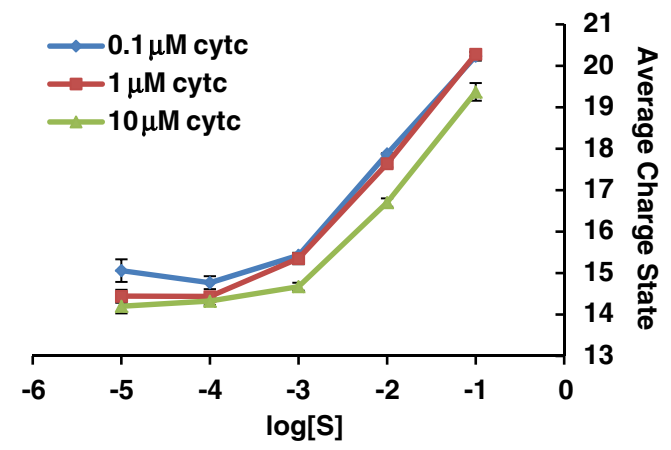

Figure 2. The average charge state of three cyt $c$ concentrations in 50:50:0.1 MeOH: $\mathrm{H}_{2} \mathrm{O}: \mathrm{FA}$ for a wide range of added sulfolane (log[S]). Irrespective of protein concentration, supercharging only became apparent at sulfolane concentrations above $10^{-3} \mathrm{M}$ the concentration of sulfolane largely independently of the concentration of the protein, at least over the range of 0.1 to $10 \mu \mathrm{M}$ cyt $c$. However, the concentration of sulfolane is $10^{3}$ to $10^{5}$ times higher than that of the protein and thus present in large molar excess.

It has been proposed that an increase in surface tension is the origin of supercharging for denatured proteins [9, 31]. However, several groups have also reported that supercharging appears to be independent of surface tension in the absence of other effects such as denaturing [23-25, 46]. One way to test the effect of surface tension on supercharging is to compare supercharging in the positive and negative modes. Very few studies of supercharging in the negative mode have been reported [31, 49]. Some supercharging reagents such as glycerol, which increase charging in the positive mode, have little to no effect in the negative mode [49], or even reduce charging [31]. Here, we also studied the effect of sulfolane on supercharging in the positive and negative modes for cyt $c$ (Figure 3). Ten $\mu \mathrm{M}$ cyt $c$ with and without $100 \mathrm{mM}$ sulfolane was prepared in 96:4 MeOH: $\mathrm{H}_{2} \mathrm{O}$, a solvent system suitable for positive and negative modes. Cyt $c$ has 24 basic sites including the N-terminus and 13 acidic sites including the C-terminus. Without sulfolane added, the spectra of cyt $c$ in the positive (Figure 3a) and negative (Figure 3b) modes reflect this difference in available charge sites with a highest intensity charge state (HICS) of +12 in the positive mode and a HICS of -7 in the negative mode. Upon addition of sulfolane, the HICS in the positive mode shifts to +15 (Figure $3 \mathrm{c}$ ) while no change in the HICS in the negative mode was observed (Figure $3 \mathrm{~d}$ ). Several adducts were observed in the negative mode, corresponding to losses of $44 \mathrm{Da}$ as well as water and methanol adducts (Online Resource 1, S1). This disparity for positive and negative modes is not consistent with surface tension being responsible for supercharging of proteins from denaturing solutions. If an increase in surface tension is the cause of supercharging, then it should occur in both the positive and negative modes since surface tension's effect on the Rayleigh limit is independent of the polarity of the charge [1].

It was noted that high concentrations of sulfolane result in protein spectra abundant with adducts. To study these adducts, the instrumental conditions were selected to minimize adduct loss. Figure 4 contains several plots of intensity against charge state for samples of cyt $c$ with various additives. Without the addition of supercharging reagent, a series of low intensity peaks corresponding to cyt $c$ with one or more adducts was observed (Figure 4a). Upon deconvolution, these peaks were assigned a mass of 97. It is known that protein solutions are prone to contain sulfate, a remnant of the protein purification process [56]. When the intensity of the protein with sulfate adducts is plotted for each charge state, it can be seen that the CSD of the adducted series follows very closely to that of the CSD of the protein without adducts, but shifted one charge state lower. These adducts are thus most likely chargeneutralizing hydrogen sulfate. No hydrogen sulfate adduct was observed on the HOCS. During supercharging, these adducts were observed to increase in intensity concurrently 

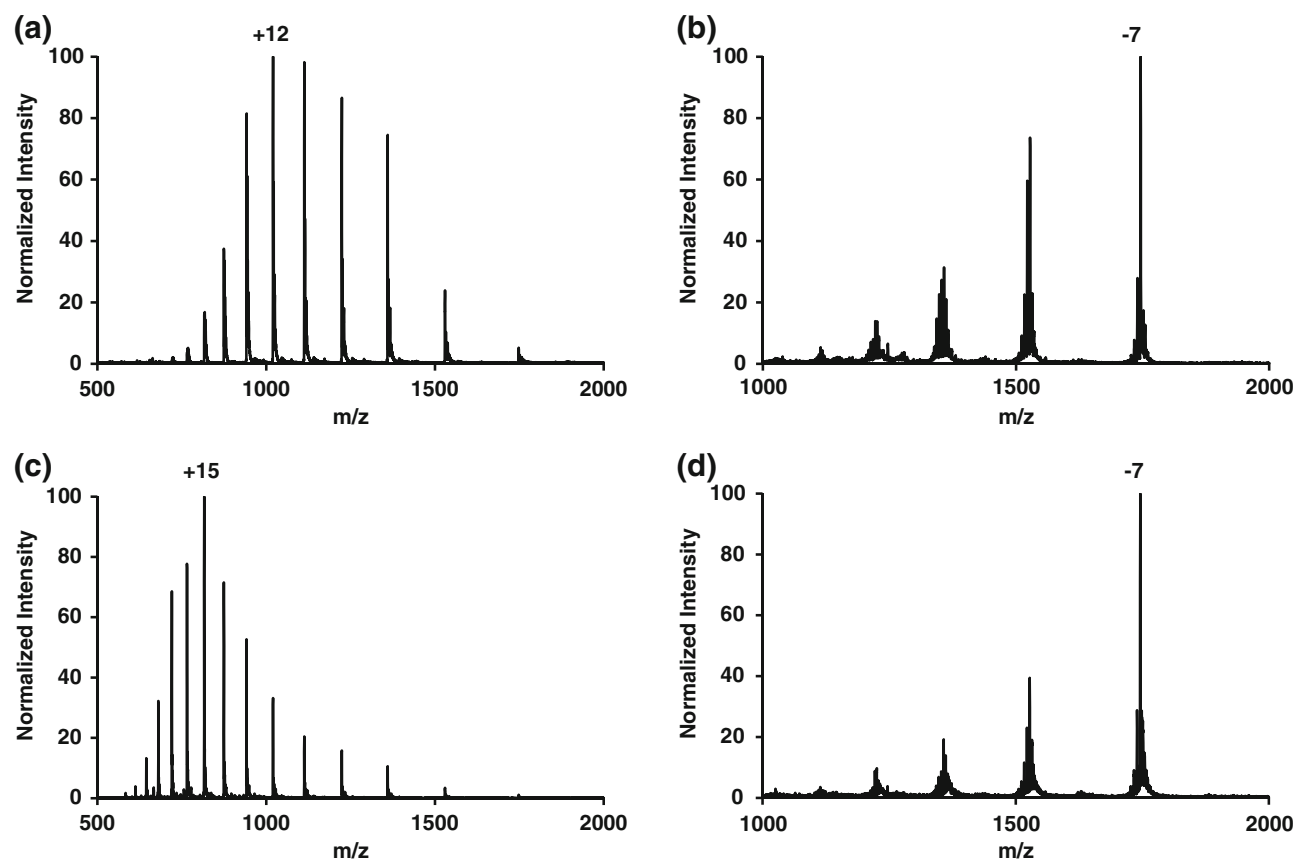

Figure 3. A comparison of positive and negative ESI-MS of $10 \mu \mathrm{M}$ cyt $c$ in $96: 4 \mathrm{MeOH}: \mathrm{H}_{2} \mathrm{O}$ with and without $100 \mathrm{mM}$ sulfolane added. (a) and (b) are positive and negative mode spectra, respectively, with no sulfolane added. (c) and (d) are positive and negative mode spectra, respectively, with sulfolane added. Supercharging upon sulfolane addition was only observed in the positive mode

(a)

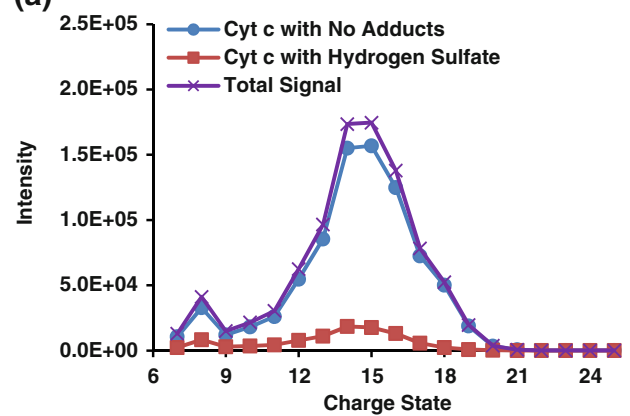

(c)

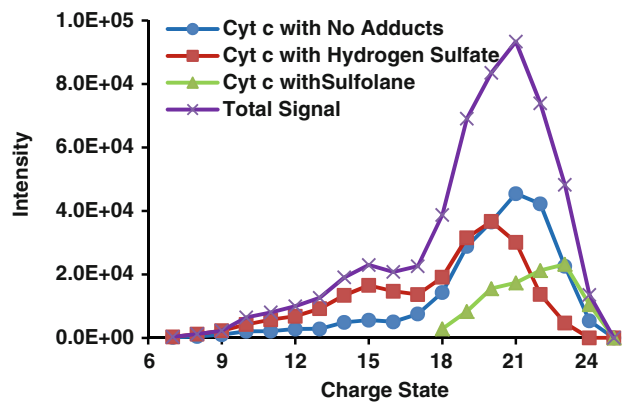

(b)

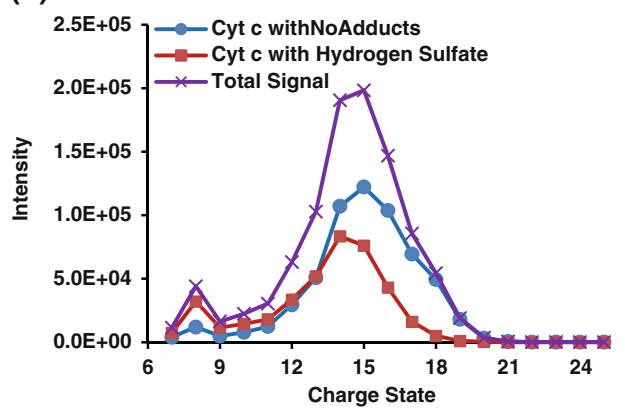

(d)

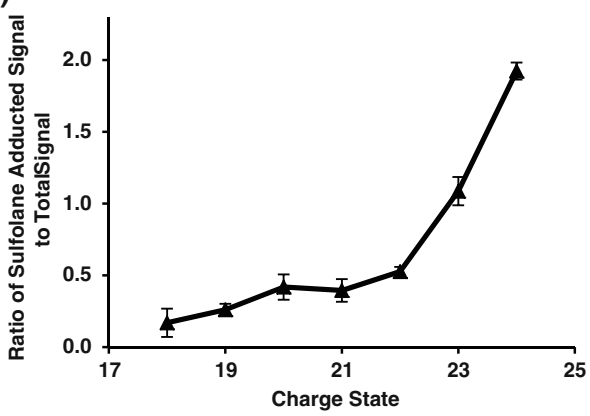

Figure 4. Spectra of cyt $c$ in in 50:50:0.1 MeOH: $\mathrm{H}_{2} \mathrm{O}: \mathrm{FA}$ with various additives. (a) Cyt $c$ with no supercharging. Hydrogen sulfate adducts were observed at low intensity. (b) Cyt $c$ with $30 \mathrm{nM}$ sulfuric acid added. No supercharging was observed although hydrogen sulfate adduction increased dramatically. (c) Cyt $c$ with $300 \mathrm{mM}$ sulfolane. Hydrogen sulfate adducts were observed with much greater intensity than in the blank and sulfolane adducts were observed for the higher charge states. (d) The ratio of the sulfolane adducted signal to the total signal for cyt $c$ plotted against the charge state. The error bars represent one standard deviation of five replicate analyses. Sulfolane adduction was seen to dramatically increase with increasing charge state 
with increasing sulfolane concentration. Additional sulfate may be produced by the oxidation of sulfolane at the positive electrode (the electrospray capillary in positive mode) to sulfur dioxide and subsequently to the sulfate ion [57]. Supercharging, hydrogen sulfate adduct formation, and signal suppression were observed to increase simultaneously upon increasing concentration of sulfolane (Figure 1b). A sample of cyt $c$ was run containing $30 \mathrm{nM}$ sulfuric acid directly as an additive (Figure $4 \mathrm{~b}$ ). No supercharging was observed for any concentration of sulfuric acid, although hydrogen sulfate adduct formation increased remarkably with additional sulfuric acid. The result confirms that hydrogen sulfate presence and adduct formation is not significant to the supercharging process.

While closely inspecting the samples containing high concentrations of sulfolane (e.g., Figure 1b), another series of adducts was observed. Unlike hydrogen sulfate, these adducts were only observed on higher charge states. A plot of the intensity of the adducted and nonadducted peaks is shown in Figure 4c. Deconvolution of these adducts revealed a mass difference of 120 , corresponding to the mass of sulfolane. Adducts of supercharging reagents were previously reported for high charge states of supercharged proteins [33, 46]. Sulfolane adducts were present even up to the +24 peak of the protein $\mathrm{CSD}$, which is the HOCS for cyt $c$ during the present study. Bovine cyt $c$ has 24 basic residues; two arginines, three histidines, 18 lysines, and the n-terminus. Since no charging beyond +24 was observed in the present system, even under highly-acidic conditions, it seems plausible that the amount of attainable supercharging may be limited by the number of basic residues present on the denatured protein available for protonation. Thus, sulfolane adducts are observed for the highest possible charge state for cyt $c$ in the present system. When the ratio of the sum of the intensities of these sulfolane adducts to the total signal of the corresponding charge state is plotted against the charge state, it can be seen that the most adduct formation occurs for the higher charge states and decreases as the charge state is decreased (Figure 4d). Sulfolane adducts are weakly bound by noncovalent interactions and are usually lost before detection.

Molecular modeling experiments have successfully predicted the charge states of proteins, including cyt $c$, observed in ESI-MS by calculating the apparent basicity of the protein in the gas phase $[18,19,58]$. Charges were assigned one at a time to the residues with the highest basicities, where the basicities of the residues were recalculated following each charge assignment. In the charge assignments reported by Peschke et al., only two charges were assigned within five residues of another charged site within the first 15 charges [19]. The first two charges were assigned to the two arginine residues while 12 of the remaining 13 were assigned to lysine residues. These results support that charge assignment is heavily directed by electrostatic interactions, since the 18 lysine residues are differentiated only by their location along the peptide chain and their proximity to other charged sites. While cyt $c$ was considered in the native state for their investigation, it is expected that this trend should also apply to the denatured state.
Our experimental results for denatured cyt $c$ appear to be consistent with electrostatics-driven charging. Figure 5 shows the amino acid sequence for bovine cyt $c$ with the basic residues highlighted. There are 14 distinct groups of single or proximally located basic sites where a charged basic site is at least four residues from another charged site. If a basic site within a group is charged, the electrostatic barrier for the charging of proximal basic residues within the same group will be elevated. The average charge state observed during ESI-MS with no supercharging reagent added was between +14 and +15 . Thus, it appears that a single charge per group is the most probable state in the typical denaturing solvent system. Using this approach, the HICS for several other denatured proteins were also successfully predicted (Online Resource 1, S2-S4).

When a supercharging reagent is added, the HICS increases to a level where multiple basic amino acids within a grouping have become charged. Concurrently, sulfolane adducts are observed to increase dramatically with increasing charge state. One possible explanation is that supercharging occurs through direct interactions between the supercharging reagent and charged basic sites. It has been demonstrated computationally that polar solvents are capable of diffusing charges through large-scale dipole ordering [59]. Solvent molecules align around a charge such that the ends with the partial charges opposite that of the charged analyte are directed towards the charge. In this way, the partial opposing charges of the solvent molecules effectively neutralize some portion of the charge on the analyte, where that portion of charge is now distributed along the periphery of this ordered solvation shell. It is supposed that several of these ordered solvation shells can form around a single charge, spreading the charge across a considerable distance. If sulfolane behaves in a similar fashion by delocalizing the charges on basic sites, the electrostatic effect on proximal basic sites could likely be reduced. Like many other identified supercharging reagents [33], sulfolane is highly polar with a dipole moment of $4.35 \mathrm{D}$, much higher than water's $1.85 \mathrm{D}$ or methanol's $1.70 \mathrm{D}$.

To test the effect of reagent polarity on supercharging ability, the dipole moments of several reagents, structurally

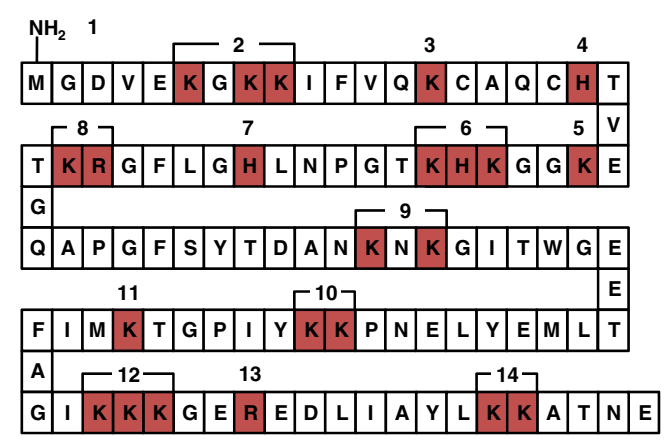

Figure 5. Amino acid sequence for bovine cyt $c$. Basic amino acids are highlighted in red. Brackets and numbers indicate groups expected to have a single charge under denaturing conditions in the absence of supercharging 
related to sulfolane, were compared with the average charge state obtained by cyt $c$ upon addition of each reagent. This information is summarized in Table 2. Each sample consisted of $10 \mu \mathrm{M}$ cyt $c$ with $100 \mathrm{mM}$ supercharging reagent in 50:50:0.1 MeOH: $\mathrm{H}_{2} \mathrm{O}: \mathrm{FA}$. Since the dipoles of all the supercharging reagents were not available in the literature, each dipole was calculated in Gaussian03W from an optimized molecular structure using the B3LYP method with a 6-311+g $(\mathrm{d}, \mathrm{p})$ basis set. The B3LYP method was chosen for its comparatively good dipole calculations achieved at a relatively low computational cost [60]. For compounds with known dipoles, a consistent overestimation averaging $16 \%$ was observed. A clear trend of increasing supercharging ability with increasing dipole moment is shown in Figure 6. Both the ACS and the HOCS increase with increasing dipole moment of the supercharging reagent.

With $\mathrm{R}^{2}$ values of 0.74 for the HOCS and 0.76 for the ACS, the regression lines in Figure 6 demonstrate that even though reagent polarity is clearly correlated with supercharging, other factors likely also contribute to supercharging ability. The interaction of supercharging reagents with charged basic sites would depend on molecular structure, such as the type and location of functional groups. For example, the most effective supercharging reagents have one or more carbonyl, sulfinyl, sulfonyl, or nitro groups present in their structure, which may be important for intermolecular interactions between the supercharging reagent and the charged basic site. Lomeli et al. demonstrated that for nitrobenzyl alcohol, supercharging increases in order of the para, meta, and ortho isomers [33]. Interestingly, this is also the order of increasing dipole moment and decreasing distance between the hydroxyl and nitro groups for this series of compounds. In the present study, sulfolene was not as effective of a supercharging reagent as sulfolane, even though their calculated dipoles were nearly identical. The presence of the double bond on sulfolene may impact how this molecule interacts with charge sites as well as other sulfolene

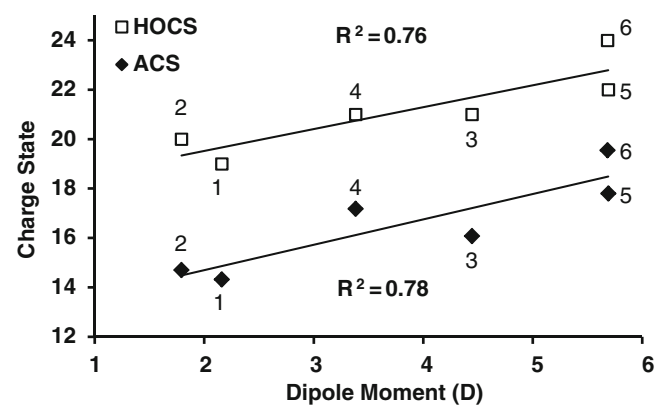

Figure 6. A plot of the HOCS and ACS of cyt $c$ in 50:50:0.1 $\mathrm{MeOH}: \mathrm{H}_{2} \mathrm{O}: \mathrm{FA}$ obtained with several supercharging reagents against their respective dipole moments. Refer to Table 2 for the reagent number key. There is a clear trend of increasing supercharging ability with increasing dipole moment. A plot containing both calculated and literature values is available in the supplemental information (Online Resource 1, S11)

molecules. Intermolecular interactions might also explain the disparity of supercharging in the positive and negative modes. Functional groups capable of interacting favorably with positively charged sites may not interact favorably with negatively charged sites. All reagents were also analyzed at the same initial concentration but display a wide range of boiling points. Differences in evaporation rates may lead to different concentrations of the supercharging reagent at the time of ion production.

\section{Conclusion}

The addition of sulfolane to denatured solutions of cyt $c$ can lead to a drastic increase of multiple charging. Upon comparing sulfolane's effect on cyt $c$ in both the positive and negative modes and confirming that supercharging only occurs in the positive mode, it seems that a more direct interaction between sulfolane and cyt $c$, other than denatur-

Table 2. Chemical Properties of Several Reagents and the ACS and HOCS for cyt $c$ Obtained in ESI-MS Upon Their Addition to a Denaturing Solution. $\mathrm{T}_{\mathrm{b}}=$ boiling point; $\mu=$ experimental dipole; $\mu^{*}=$ calculated dipole. A “-” Indicates that no Data could be Found. Supercharged Spectra of cyt $c$ for these Additional Compounds can be Found in the Online Resource 1 (S5-S10)

\begin{tabular}{|c|c|c|c|c|c|}
\hline Reagent & $\mathrm{T}_{\mathrm{b}}\left({ }^{\circ} \mathrm{C}\right)$ & $\mu(D)$ & $\mu^{*}(\mathrm{D})$ & ACS & HOCS \\
\hline 1. Water & 100 & 1.85 & 2.16 & 14.3 & 19 \\
\hline 2. Benzyl alcohol & 205 & 1.71 & 1.79 & 14.7 & 20 \\
\hline 3. Dimethyl sulfoxide & 189 & 3.96 & 4.44 & 16.1 & 21 \\
\hline 4. 3-Chlorothiete-1,1-dioxide & - & - & 3.38 & 17.2 & 21 \\
\hline 5. Sulfolene & - & - & 5.69 & 17.8 & 22 \\
\hline 6. Sulfolane & 285 & 4.35 & 5.68 & 19.6 & 24 \\
\hline
\end{tabular}


ing or increased charge availability, could be responsible for supercharging in this system. This claim is further supported by the strong relationship between the degree of reagent adduct formation and the enhancement of multiple charging. Since the presence of adducts is observed to increase concomitantly with increasing charge state, they appear to be important to the supercharging process. We propose that through a direct interaction of sulfolane with charged basic sites a means is provided for basic sites, proximal to existing charges, to overcome the electrostatic barrier and obtain or retain charges. The exact details of this possible mechanism are currently under investigation, but we believe the stabilization is provided through charge delocalizing through large scale solvent reordering away from the basic site due to the high dipole moment of sulfolane and other supercharging reagents. The clear correlation between the dipole moments of several supercharging reagents and the extent of supercharging observed for cyt $c$ supports this claim.

\section{Acknowledgments}

The authors thank Western Michigan University for funding and John B. Miller for his useful discussion.

\section{References}

1. Rayleigh, L.: On the equilibrium of liquid conducting masses charged with electricity. Philos. Mag. 14, 184-186 (1882)

2. Cole, R.B.: Some tenets pertaining to electrospray ionization mass spectrometry. J. Mass Spectrom. 35, 763-772 (2000)

3. Taflin, D.C., Ward, T.L., Davis, E.J.: Electrified droplet fission and the Rayleigh limit. Langmuir 5, 376-384 (1989)

4. Iribarne, J.V., Thomson, B.A.: On the evaporation of small ions from charged droplets. J. Chem. Phys. 64, 2287-2294 (1976)

5. Thomson, B.A., Iribarne, J.V.: Field induced ion evaporation from liquid surfaces at atmospheric pressure. J. Chem. Phys. 71, 4451-4463 (1979)

6. Tang, L., Kebarle, P.: Dependence of ion intensity in electrospray mass spectrometry on the concentration of the analytes in the electrosprayed solution. Anal. Chem. 65, 3654-3668 (1993)

7. Dole, M., Mack, L.L., Hines, R.L., Mobley, R.C., Ferguson, L.D., Alice, M.B.: Molecular beams of Mmacroions. J. Chem. Phys. 49, 2240-2249 (1968)

8. Fernandez de la Mora, J.: Electrospray ionization of large multiply charged species proceeds via Dole's charged residue mechanism. Anal. Chim. Acta 406, 93-104 (2000)

9. Iavarone, A.T., Williams, E.R.: Mechanism of charging and supercharging molecules in electrospray ionization. J. Am. Chem. Soc. 125, 2319-2327 (2003)

10. Kaltashov, I.A., Mohimen, A.: Estimates of protein surface areas in solution by electrospray ionization mass spectrometry. Anal. Chem. 77, 5370-5379 (2005)

11. Gamero-Castaño, M., Fernández de la Mora, J.: Kinetics of small ion evaporation from the charge and mass distribution of multiply charged clusters in electrosprays. J. Mass Spectrom. 35, 790-803 (2000)

12. Gamero-Castaño, M., Fernández de la Mora, J.: Mechanisms of electrospray ionization of singly and multiply charged salt clusters. Anal. Chim. Acta 406, 67-91 (2000)

13. Loo, J.A., Udseth, H.R., Smith, R.D., Futrell, J.H.: Collisional effects on the charge distribution of ions from large molecules, formed by electrospray-ionization mass spectrometry. Rapid Commun. Mass Spectrom. 2, 207-210 (1988)

14. Covey, T.R., Bonner, R.F., Shushan, B.I., Henion, J., Boyd, R.K.: The determination of protein, oligonucleotide and peptide molecular weights by ion-spray mass spectrometry. Rapid Commun. Mass Spectrom. 2 , 249-256 (1988)
15. Wong, S.F., Meng, C.K., Fenn, J.B.: Multiple charging in electrospray ionization of poly(ethylene glycols). J. Phys. Chem. 92, 546-550 (1988)

16. Schnier, P., Gross, D., Williams, E.: On the maximum charge state and proton transfer reactivity of peptide and protein ions formed by electrospray ionization. J. Am. Soc. Mass Spectrom. 6, 1086-1097 (1995)

17. Konermann, L.: A Minimalist model for exploring conformational effects on the electrospray charge state distribution of proteins. J. Phys. Chem. B 111, 6534-6543 (2007)

18. Williams, E.R.: Proton transfer reactivity of large multiply charged ions. J. Mass Spectrom. 31, 831-842 (1996)

19. Peschke, M., Blades, A., Kebarle, P.: Charged states of proteins. Reactions of doubly protonated alkyldiamines with $\mathrm{NH}_{3}$ : solvation or deprotonation. Extension of two proton Cases to multiply protonated globular proteins observed in the gas phase. J. Am. Chem. Soc. 124, 11519-11530 (2002)

20. Fenselau, C., Szilagyi, Z., Williams, T.: Intercharge distances in $\mathrm{Zn}_{7^{-}}$ metallothionein analyzed by nanospray on a quadrupole ion trap and molecular modeling. J. Mass Spectrom. Soc. Jpn. 48, 23-25 (2000)

21. Heck, A.J.R., van den Heuvel, R.H.H.: Investigation of intact protein complexes by mass spectrometry. Mass Spectrom. Rev. 23, 368-389 (2004)

22. Nesatyy, V.J., Suter, M.J.F.: On the conformation-dependent neutralization theory and charging of individual proteins and their noncovalent complexes in the gas phase. J. Mass Spectrom. 39, 93-97 (2004)

23. Samalikova, M., Matecko, I., Muller, N., Grandori, R.: Interpreting conformational effects in protein nano-ESI-MS spectra. Anal. Bioanal. Chem. 378, 1112-1123 (2004)

24. Samalikova, M., Grandori, R.: Testing the role of solvent surface tension in protein ionization by electrospray. J. Mass Spectrom. 40, 503-510 (2005)

25. Samalikova, M., Grandori, R.: Protein charge-state distributions in electrospray-ionization mass spectrometry do not appear to be limited by the surface tension of the solvent. J. Am. Chem. Soc. 125, 13352-13353 (2003)

26. Katta, V., Chait, B.T.: Observation of the heme-globin complex in native myoglobin by electrospray-ionization mass spectrometry. J. Am. Chem. Soc. 113, 8534-8535 (1991)

27. Grandori, R.: Origin of the conformation dependence of protein chargestate distributions in electrospray ionization mass spectrometry. J. Mass Spectrom. 38, 11-15 (2003)

28. Testa, L., Brocca, S., Grandori, R.: Charge-surface correlation in electrospray ionization of folded and unfolded proteins. Anal. Chem. 83, 6459-6463 (2011)

29. Wang, G., Cole, R.B.: Effects of solvent and counterion on ion pairing and observed charge states of diquaternary ammonium salts in electrospray ionization mass spectrometry. J. Am. Soc. Mass Spectrom. 7, 1050-1058 (1996)

30. Mirza, U.A., Chait, B.T.: Effects of anions on the positive ion electrospray ionization mass spectra of peptides and proteins. Anal. Chem. 66, 2898-2904 (1994)

31. Iavarone, A.T., Williams, E.R.: Supercharging in electrospray ionization: effects on signal and charge. Int. J. Mass Spectrom. 219, 63-72 (2002)

32. Iavarone, A.T., Jurchen, J.C., Williams, E.R.: Supercharged protein and peptide Ions formed by electrospray ionization. Anal. Chem. 73, 1455-1460 (2001)

33. Lomeli, S.H., Peng, I.X., Yin, S., Ogorzalek Loo, R.R., Loo, J.A.: New reagents for increasing ESI multiple charging of proteins and protein complexes. J. Am. Soc. Mass Spectrom. 21, 127-131 (2010)

34. Chowdhury, S.K., Katta, V., Chait, B.T.: Probing conformational changes in proteins by mass spectrometry. J. Am. Chem. Soc. 112, 9012-9013 (1990)

35. Loo, J.A., Loo, R.R.O., Udseth, H.R., Edmonds, C.G., Smith, R.D.: Solvent-induced conformational changes of polypeptides probed by electrospray-ionization mass spectrometry. Rapid Commun. Mass Spectrom. 5, 101-105 (1991)

36. Blanc, J.C.Y.L., Beuchemin, D., Siu, K.W.M., Guevremont, R., Berman, S.S.: Thermal denaturation of some proteins and its effect on their electrospray mass spectrat. Org. Mass Spectrom. 26, 831-839 (1991)

37. Kharlamova, A., Prentice, B.M., Huang, T.-Y., McLuckey, S.A.: Electrospray droplet exposure to gaseous acids for the manipulation of protein charge state distributions. Anal. Chem. 82, 7422-7429 (2010) 
38. McLuckey, S.A., Van Berkel, G.J., Glish, G.L.: Reactions of dimethylamine with multiply charged ions of cytochrome c.J. Am. Chem. Soc. 112, 5668$5670(1990)$

39. Winger, B.E., Light-Wahl, K.J., Richard, D.S.: Gas-phase proton transfer reactions involving multiply charged cytochrome $c$ ions and water under thermal conditions. J. Am. Soc. Mass Spectrom. 3, 624-630 (1992)

40. Fenn, J.B.: Ion formation from charged droplets: roles of geometry, energy, and time. J. Am. Soc. Mass Spectrom. 4, 524-535 (1993)

41. Thomson, B.A.: Declustering and fragmentation of protein ions from an electrospray ion source. J. Am. Soc. Mass Spectrom. 8, 1053-1058 (1997)

42. Li, Y., Cole, R.B.: Shifts in peptide and protein charge state distributions with varying spray tip orifice diameter in nanoelectrospray Fourier transform ion cycitron resonance mass spectrometry. Anal. Chem. 75, 5739-5746 (2003)

43. Iavarone, A.T., Williams, E.R.: Collisionally activated dissociation of supercharged proteins formed by electrospray ionization. Anal. Chem. 75, 4525-4533 (2003)

44. Yin, S., Loo, J.A.: Top-down mass spectrometry of supercharged native protein-ligand complexes. Int. J. Mass Spectrom. 300, 118-122 (2011)

45. Iavarone, A.T., Jurchen, J.C., Williams, E.R.: Effects of solvent on the maximum charge state and charge state distribution of protein ions produced by electrospray ionization. J. Am. Soc. Mass Spectrom. 11, 976-985 (2000)

46. Lomeli, S.H., Yin, S., Ogorzalek Loo, R.R., Loo, J.A.: Increasing charge while preserving noncovalent protein complexes for ESI-MS. $J$. Am. Soc. Mass Spectrom. 20, 593-596 (2009)

47. Sterling, H.J., Williams, E.R.: Origin of supercharging in electrospray ionization of noncovalent complexes from aqueous solution. J. Am. Soc. Mass Spectrom. 20, 1933-1943 (2009)

48. Sterling, H., Prell, J., Cassou, C., Williams, E.: Protein conformation and supercharging with DMSO from aqueous solution. J. Am. Soc. Mass Spectrom. 22, 1178-1186 (2011)

49. Sterling, H., Daly, M., Feld, G., Thoren, K., Kintzer, A., Krantz, B., Williams, E.: Effects of supercharging reagents on noncovalent complex structure in electrospray ionization from aqueous solutions. J. Am. Soc. Mass Spectrom. 21, 1762-1774 (2010)
50. Sterling, H.J., Williams, E.R.: Real-time hydrogen/deuterium exchange kinetics via supercharged electrospray ionization tandem mass spectrometry. Anal. Chem. 82, 9050-9057 (2010)

51. Hogan Jr., C.J., Ogorzalek Loo, R.R., Loo, J.A., Fernández de la Mora, J.: Ion mobility-mass spectrometry of phosphorylase B ions generated with supercharging reagents but in charge-reducing buffer. Phys. Chem. Chem. Phys. 12, 13476-13483 (2010)

52. Šamalikova, M., Matečko, I., Müller, N., Grandori, R.: Interpreting conformational effects in protein nano-ESI-MS spectra. Anal. Bioanal. Chem. 378, 1112-1123 (2004)

53. Wang, G.D., Cole, R.B.: Effect of solution ionic-strength on analyte charge-state distributions in positive and negative-ion electrospray mass-spectrometry. Anal. Chem. 66, 3702-3708 (1994)

54. Babu, K.R., Moradian, A., Douglas, D.J.: The methanol-induced conformational transitions of beta-lactoglobulin, cytochrome $c$, and ubiquitin at low pH: A study by electrospray ionization mass spectrometry. J. Am. Soc. Mass Spectrom. 12, 317-328 (2001)

55. Kamatari, Y.O., Konno, T., Kataoka, M., Akasaka, K.: The Methanolinduced globular and expanded denatured states of cytochrome $c$ : a Study by CD fluorescence, NMR, and small-angle X-ray scattering. $J$. Mol. Biol. 259, 512-523 (1996)

56. Chowdhury, S.K., Katta, V., Beavis, R.C., Chait, B.T.: Origin and removal of adducts (molecular mass $=98 \mathrm{u}$ ) attached to peptide and protein ions in electrospray ionization mass spectra. J. Am. Soc. Mass Spectrom. 1, 382-388 (1990)

57. Wasmus, S., Vielstich, W.: Electrooxidation and reduction of dimethyl sulfoxide and sulfolane in aqueous acid solution. An on-line MS study. Electrochim. Acta 38, 175-183 (1993)

58. Touboul, D., Jecklin, M.C., Zenobi, R.: Investigation of deprotonation reactions on globular and denatured proteins at atmospheric pressure by ESSI-MS. J. Am. Soc. Mass Spectrom. 19, 455-466 (2008)

59. Ahadi, E., Konermann, L.: Surface charge of electrosprayed water nanodroplets: a molecular dynamics study. J. Am. Chem. Soc. 132, 11270-11277 (2010)

60. Cramer, C.J.: Essentials of Computational Chemistry, 2nd edn. Wiley, Chichester, England (2004) 